\title{
Quadriceps Muscle Fatigue and Knee Joint Position Sense in Healthy Men
}

\author{
Mohammad Mohammadi Bazneshin ${ }^{1}$, Ali Amiri ${ }^{1 *}$, Ali Ashraf Jamshidi' ${ }^{1}$ Behnoosh Vasaghi-Gharamaleki² \\ 1. Department of Physiotherapy, School of Rehabilitation Sciences, Iran University of Medical Sciences, Tehran, Iran. \\ 2. Department of Basic Sciences, School of Rehabilitation Sciences, Iran University of Medical Sciences, Tehran, Iran.
}

Citation: Mohammadi Bazneshin M, Amiri A, Jamshidi AA, Vasaghi-Gharamaleki B. Quadriceps muscle fatigue and knee joint position sense in healthy men. Physical Therapy. 2015; 5(2):109-114. http://dx.crossref.org/10.15412/J.PTJ.07050207

: http://dx.crossref.org/10.15412/J.PTJ.07050207

Article info:

Received: 30 Jan. 2015

Accepted: 28 Apr. 2015

Keywords:

Knee, Proprioception, Fatigue, Quadriceps muscle

\begin{abstract}
A B S T RA C T
Purpose: Proprioception is a part of the somatosensory system responsible for sending information to the central nervous system to monitor the status of various body parts relative to each another. One of the main causes of impaired proprioception is fatigue. In the following study, the effects of fatigue on quadriceps muscle and hence on knee proprioception in healthy subjects was studied.

Methods: This is a pretest-posttest study. By available sampling, 34 healthy young subjects with a mean (SD) age of 22.15 (1.97) years, a normal knee motion range and muscle strength, with no skeletal muscle nerve damage, volunteered for this study. The subjects tested the target angle reconstruction ( $45^{\circ}$ flexion) of their knee joints while contracting the quadriceps muscles. We used a digital inclinometer to assess proprioception before and after fatigue. Then, by using the paired t-test, the rate of constant error and absolute error were compared. For the statistical analyses, SPSS (version 16) was used.
\end{abstract}

Results: quadriceps muscle fatigue causes a significant increase in the error rate of the knee joint reconstruction angle at $45^{\circ}$ (absolute error and constant error) $(\mathrm{P}<0.05)$.

Conclusion: Quadriceps muscle fatigue decreases accuracy and increases absolute error and constant reconstruction angle of the knee joint in healthy individual.

\section{Introduction}

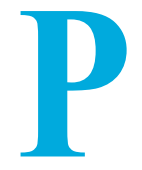

roprioception is a part of the somatosensory system, responsible for collecting information and sending it to the central nervous system to monitor the status of various body parts relative to each another $[1,2]$.
Proprioception is also responsible for controlling neuromuscular signals and processing information from stimuli to the central nervous system (CNS), as well as receiving signals from afferent neurons [2]. joint position sense is an important factor in joint coordination, muscle stiffness and integrated movement and Disruption of it could lead to injury [3]. Several factors can disrupt proprioception, followed by the motor control dysfunction and abnormal load on the joints.

\footnotetext{
* Corresponding Author:

Ali Amiri, PhD

Address: Department of Physiotherapy, School of Rehabilitation Sciences, Iran University of Medical Sciences, Tehran, Iran.

Phone: +98 (911) 3949202

E-mail amiri.alipt10@yahoo.com
} 
One of these factors is fatigue $[4,5]$. The ability to work or performing sports activities during muscle fatigue is of great importance. The majority of injuries inflicted on athletes occur at the end of the exercise, and especially in their lower extremities [6]. Muscle fatigue is the loss of the capacity of muscle power generation during voluntary contraction [7]. Exercising to fatigue may change subjects' reproduction ability of knee joint angles. This may represent a decline in proprioceptive function after heavy exercise bouts [8].

Analysing the joint position sense is one of the most common test to assess proprioception. The accuracy of the reconstruction of the joint angle is measured by active and passive joint angles or by evaluating and carefully considering the subjects joint angle movement abilities [9]. So far several studies have examined the effect of muscle fatigue on proprioception of the joints. Some studies reported that fatigue impairs proprioception $[1,8,10,11]$, and some reported no effect [12-14]. Therefore, conflicting results have been found in this field. Different kinds of fatigue, their mechanisms and different effects on proprioception in previous studies indicate the need for further research.

The knee is the one of the most complex and largest joints in the body. Many people have reported injuries during sports and work activities [15]. The quadriceps muscles of the lower extremities are among the most important muscles in keeping the knee joint dynamic and stable [16]. Therefore, this study aimed to evaluate the effect of quadriceps muscle fatigue on regeneration of the knee joint angles.

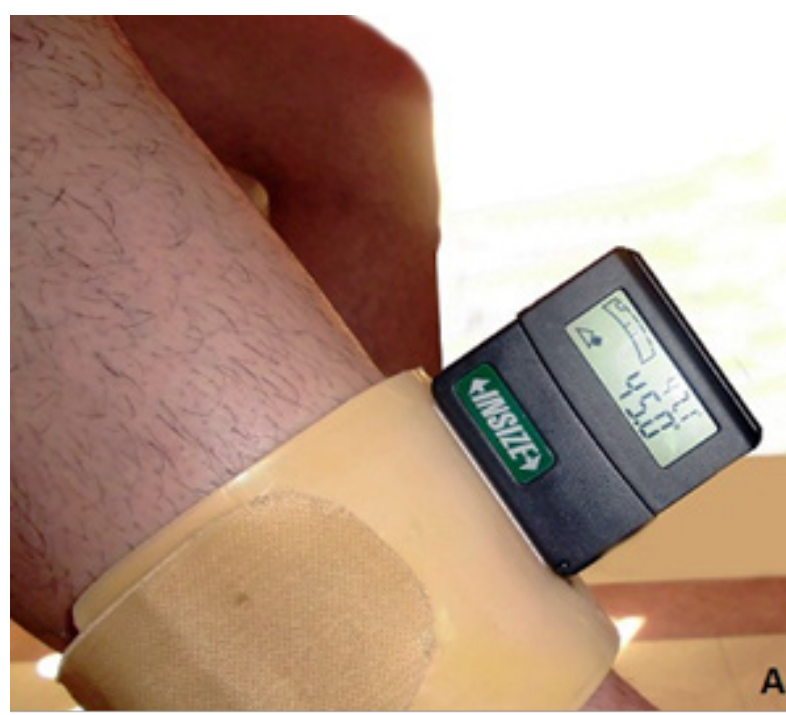

\section{Materials and Methods}

This study has pretest-posttest design and is interventional. A total of 34 healthy male volunteers using available sampling method were selected to participate in the study. Exclusion criteria included a history of lower extremity injury in the last 6 months and especially fractures in the lower extremity, surgery in the past year, as well as neuromusculoskeletal disorders (diagnosed by especial tests), vestibular disorders, and cardiovascular problems. This study was done in Tehran City in the academic year 2015-2016.

Firstly, the maximum isometric quadriceps strength of each participant was measured using a dynamometer (pullpush dynamometer-zemic $250 \mathrm{~kg}$ ). The subject would sit on a bed that was designed to be used with the dynamometer. The subject's dominant knee was positioned and flexed at $90^{\circ}$ angle. The dynamometer was placed 2 fingers above the lateral malleolus on the anterior part of the tibia. The subject was asked to apply maximum voluntary isometric contraction of quadriceps femoris muscle to the dynamometer for 3 seconds, and the force was recorded. This procedure was repeated 3 times and their average results was recorded for later comparison.

During the next phase, the errors of the joint angles reconstruction were measured. We used digital (INSIZE INC. (USA) $0^{\circ}-180^{\circ}$, accuracy of $0.1^{\circ}$ ) to measure the extent of reconstruction error of the knee angle [17]. Also a device was designed to place the inclinometer on the mid-calf of the subject. The subject's thigh was fixed on the bed. The examiner placed the subject's knee at the target angle $\left(45^{\circ}\right)$ and asked

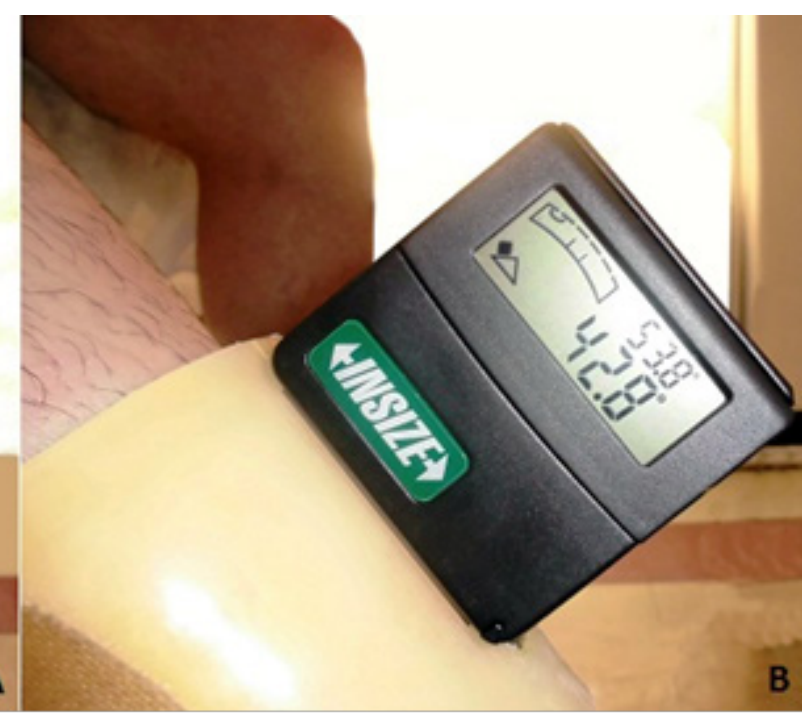

PHYSICAL TREA † MENTS

Figure 1. A. Examiner passively move the knee into target angle $\left(45^{\circ}\right)$ and ask subject to keep it for 7 seconds.

B. The subject reconstruct the angle with closed eyes. 
the subject to contract his quadriceps muscle and hold it in place for 7 seconds. The examiner then returned the subject's knee to its original position and asked him to close his eyes and reconstruct the target angle by moving the knee from the original $90^{\circ}$ angle towards the target $45^{\circ}$ angle.

Subsequently, the fatigue protocol was put in place. The subject sat on a chair; a weight which was $70 \%$ of the maximum quadriceps isometric force was tied around the subject's leg. The subject was asked, to the extent of his ability, repeatedly perform the knee extension range of motion until he was no longer able to execute the move. Then, a weight which was $50 \%$ of maximum isometric force of the quadriceps was tied and the subject was asked to repeat the fatigue protocol to the best of his ability. The movements were done in such a way that each contraction cycle lasted 8 seconds (4 seconds of concentric contraction and 4 seconds of eccentric contraction of the quadriceps muscle). Next, the maximum isometric force of the quadriceps was measured again and if it was $50 \%$ or less, compared to the initial obtained value, the proprioception assessment would be carried out. Otherwise, the fatigue protocol was repeated $[18,19]$. The extent of error of the knee angle reconstruction, before and after fatigue, was measured by the inclinometer (Figure 1).

To accurately evaluate proprioception, 2 variables were considered: 1) Absolute error, which measures the difference between the measured angle and the target angle without taking into consideration the direction of error, and 2) Constant error, which measures the difference between the measured angle and the target angle, taking into consideration the direction of error. False positive errors, or overes- timates, are going over the target angle, and false negative errors, or underestimates, are not reaching the target angle [20]. The fewer errors, the more accurate and stable the reconstruction would be. Using the Kolmogorov-Smirnov statistical analysis, the normality of the data were revealed. This information was used for statistical calculation of the parametric tests. In order to compare the variable of proprioception, before and after fatigue, paired sample t test was used. The significance level for all calculations in the statistical analysis was considered at $\mathrm{P}=0.05$. For the statistical calculations, SPSS (version 16) was used.

\section{Ethical considerations}

After visiting the test setting (Control Laboratory of Faculty of Rehabilitation, Iran University of Medical Sciences), the participants signed informed consent forms that have been approved by the Ethics Committee of Iran University of Medical Sciences.

\section{Results}

The demographic data included age, height, weight, and body mass index (Table 1). The absolute and constant error of the measured angle were compared before and after fatigue, which showed a statistically significant difference (absolute error, $\mathrm{P}<0.001$; constant error, $\mathrm{P}=$ 0.006) (Figure 2 and Table 2).

\section{Discussion}

The results of this study showed that the quadriceps muscle fatigue decreases the accuracy of the reconstruc-

Table 1. Demographic characteristics of the study participants.

\begin{tabular}{ccccc}
\hline & Mean & SD & Minimum & 19 \\
\hline Age $(\mathrm{y})$ & 22.15 & 1.97 & 1.65 \\
Height $(\mathrm{m})$ & 1.77 & 0.05 & 60 & 1.88 \\
Weight $(\mathrm{kg})$ & 72.85 & 6.49 & 25 \\
Body mass index $\left(\mathrm{kg} / \mathrm{m}^{2}\right)$ & 23.15 & 1.87 & 20.68 & 28.08 \\
\hline
\end{tabular}

PHYSICAL TREA $\pitchfork$ MENTS

Table 2. Comparing the absolute and constant errors of knee joint repositioning angle $\left(45^{\circ}\right)$, before and after quadriceps muscle fatigue.

\begin{tabular}{|c|c|c|c|c|c|c|}
\hline \multirow{2}{*}{ Variables } & \multicolumn{2}{|c|}{ Before fatigue } & \multicolumn{2}{|c|}{ After fatigue } & \multirow{2}{*}{$\mathbf{t}$} & \multirow{2}{*}{ Significant level } \\
\hline & Mean & SD & Mean & SD & & \\
\hline Absolute error (degree) & 1.37 & 0.39 & 4.01 & 0.89 & 19.105 & 0.000 \\
\hline Constant error (degree) & 0.44 & 1.05 & 1.96 & 2.99 & 2.961 & 0.006 \\
\hline
\end{tabular}



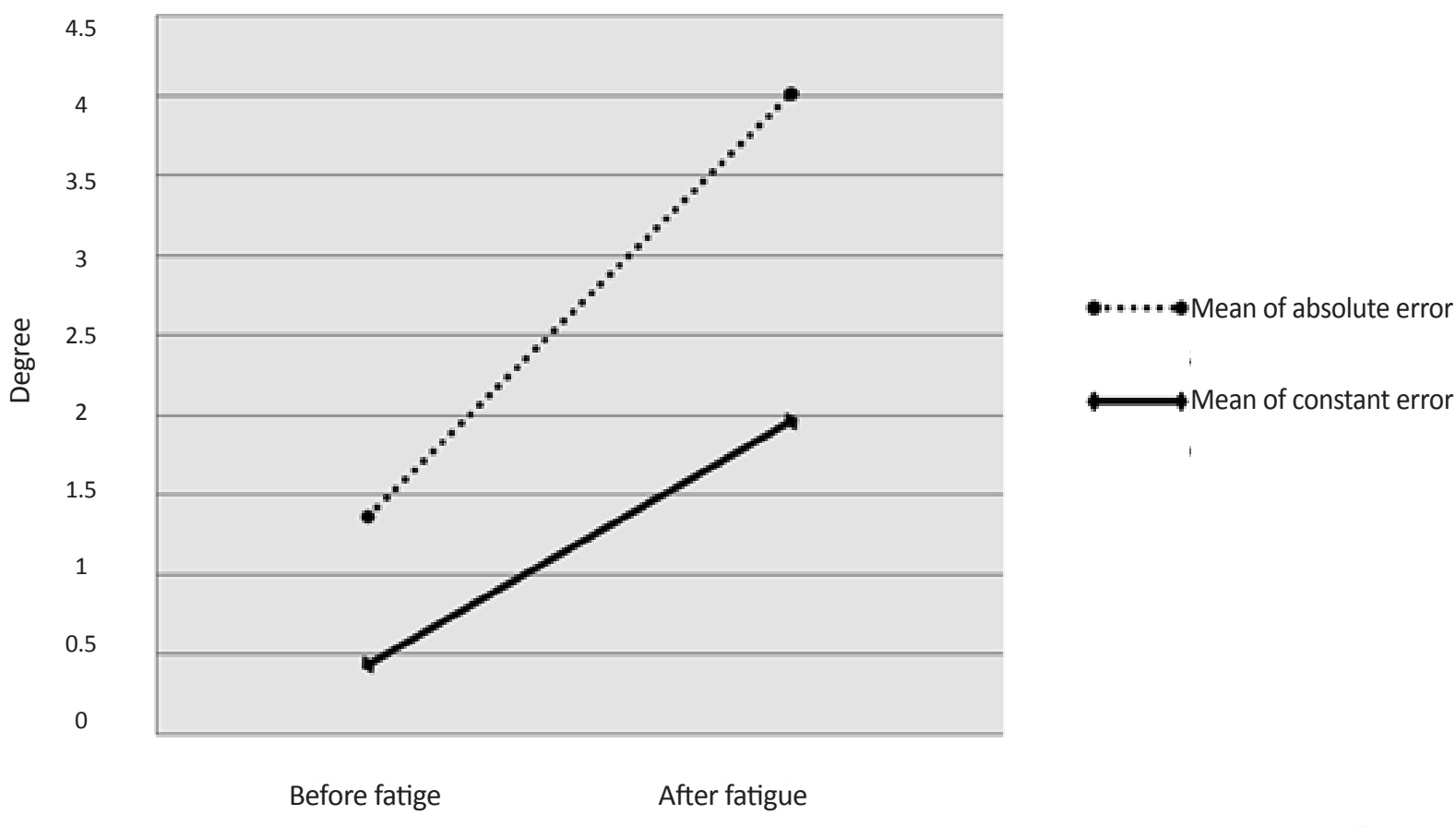

Figure 2. Comparing the absolute and constant errors of knee joint repositioning angle $\left(45^{\circ}\right)$, before and after quadriceps muscle fatigue.

tion of the joint angles in young men. Considering the role of proprioception in reconstruction of joint angles, muscle fatigue can have direct effects in this regard, which increases the risk of potential damage.

There are various reasons for this finding. Following muscle fatigue, pain receptors are activated through the production of metabolites caused by muscle contraction [21]. These metabolites directly affect the pattern of muscle spindle discharge, resulting in the reduction of muscle's afferent signals [7]. The reduction in the sensitivity of proprioceptive afferent nerves, which are responsible for relaying information to CNS, can reduce the accuracy of the reconstructed joint angle. Yaggie et al. found that the impairments in the position sense that occurred after fatigue could be due to the reduction of motor neuron output and sensitivity of the muscle afferents groups III and IV [22].

In another study conducted by Ribeiro et al. on knee proprioception of elderly individuals, the extent of absolute error of the reconstructed knee angle increased after fatigue of the quadriceps muscles. Based on these findings, they suggested that muscle fatigue, following an increase in the threshold of muscle spindle discharge can impair proprioception [23]. It can also impair the control of muscle nerves and disable muscles to maintain joint dynamic stability [24].
The study results of the Miura et al. who examined local fatigue on knee proprioception are contrary to our results [14]. In their study, local fatigue did not have any effect on knee proprioception. This difference can be due to the procedures carried out in the 2 fatigue studies. In the study conducted by Miura et al., there was no objective criterion for determining the exhaustion threshold and all study participants were equally tired. Thus, their participants might not measure up to the fatigue criteria of our study ( $50 \%$ or less than the maximum muscle strength). Furthermore, the measured target angle in Miura et al. study was randomly chosen to be between $10^{\circ}$ and $80^{\circ}$. During this range of motion in some angles, ligaments play an important and crucial role in proprioception of the knee angle, and therefore, muscle fatigue can be compensated by this factor. The measured target angle in our study $\left(45^{\circ}\right)$ is an angle that the muscle has its most share in maintaining the dynamic stability of the joint.

According to the obtained mean constant error, most people surpassed the target angle and had more positive errors (overestimate). It could be due to increase in the threshold of muscle spindle discharge According to the results of Sajjadi et al. who studied the effect of fatigue on proprioception of the neck between men and women, men showed a higher amount of positive errors compared to woman who had more negative errors [25]. This result can indicate that men do not follow the Internal Forward 
Model hypothesis (which was introduced by Givoni et al.). They stated that in the Internal Forward Model, the brain compares and integrates the predicted feedback to the actual feedback it receives from the quadriceps muscles. This comparison leads to the assumption that the quadriceps muscle is longer than its actual state and accordingly the proprioceptive error occurs [26].

The following study indicates that quadriceps muscle fatigue to the amount of $50 \%$ maximum muscle strength, will result in decrease in the accuracy and increase in reconstruction error at $45^{\circ}$ angle of the knee joint. This increase in error occurs more in the form of overestimate (surpassing the target angle) and creating more positive errors.

\section{Acknowledgements}

This study is a part of Mr. Mohammad Mohammadi Bazneshin's Master's thesis at the School of Rehabilitation, Iran University of Medical Sciences. Financially, it was supported by Iran University of Medical Sciences.

\section{Conflict of Interests}

The authors declared no conflict of interests.

\section{References}

[1] Bayramoglu M, Toprak R, Sozay S. Effects of osteoarthritis and fatigue on proprioception of the knee joint. Archives of Physical Medicine and Rehabilitation. 2007; 88(3):346-50.

[2] Biedert R. Contribution of the three levels of nervous system motor control: spinal cord, lower brain, cerebral cortex. In: Le SM, Fu FH, editors. proprioception and neuromuscular control in joint stability. Chamahgn, I.L.: Human Kinetics; 2000, p. 23-31.

[3] Madhavan S, Shields RK. Influence of age on dynamic position sense: evidence using a sequential movement task. Experimental Brain Research. 2005; 164(1):18-28.

[4] Taylor JL, Butler JE, Gandevia S. Changes in muscle afferents, motoneurons and motor drive during muscle fatigue. European Journal of Applied Physiology. 2000; 83(2-3):10615.

[5] Zwarts M, Bleijenberg G, Van Engelen B. Clinical neurophysiology of fatigue. Clinical Neurophysiology. 2008; 119(1):2-10

[6] Augustsson J, Thomeé R, Linden C, Folkesson M, Tranberg $\mathrm{R}$, Karlsson J. Single-leg hop testing following fatiguing exercise: reliability and biomechanical analysis. Scandinavian Journal of Medicine \& Science in Sports. 2006; 16(2):111-20.
[7] Sogaard K, Gandevia SC, Todd G, Petersen NT, Taylor JL. The effect of sustained low-intensity contractions on supraspinal fatigue in human elbow flexor muscles. Journal of Physiology. 2006; 573(2):511-23.

[8] Lattanizio PJ, Petrella RJ, Sproule JR, Fowler PJ. Effects of fatigue on knee proprioception. Clinical Journal of Sport Medicine. 1997; 7(1):22-7.

[9] Smith MP, Sizer PS, James CR. Effects of fatigue on frontal plane knee motion, muscle activity, and ground reaction forces in men and women during landing. Journal of Sports Science \& Medicine. 2009; 8(3):419-23.

[10] Vuillerme N, Boisgontier M. Muscle fatigue degrades force sense at the ankle joint. Gait \& Posture. 2008; 28(3):521-24.

[11] Sharpe MH, Miles TS. Position sense at the elbow after fatiguing contractions. Experimental Brain Research. 1993; 94(1):179-82.

[12] Gurney B, Milani J, Pedersen ME. Role of fatigue on proprioception of the ankle. Journal of Exercise Physiology. 2000; 3(1):8-13.

[13] South M, George KP. The effect of peroneal muscle fatigue on ankle joint position sense. Physical Therapy in Sport. 2007; 8(2):82-87.

[14] Miura K, Ishibashi Y, Tsuda E, Okamura Y, Otsuka H, Toh $\mathrm{S}$. The effect of local and general fatigue on knee proprioception. Arthroscopy. 2004; 20(4):414-18.

[15] Louw QA, Manilall J, Grimmer K. Epidemiology of knee injuries among adolescents: a systematic review. British Journal of Sports Medicine. 2008; 42(1):2-10.

[16] Houglum PA, Bertoti DB. Brunnstrom's clinical kinesiology. Philadelphia: FA Davis Company; 2011

[17] Dover G, Powers ME. Reliability of joint position sense and force-reproduction measures during internal and external rotation of the shoulder. Journal of Athletic Training. 2003; 38(4):304-10.

[18] Parijat P, Lockhart TE. Effects of quadriceps fatigue on the biomechanics of gait and slip propensity. Gait \& Posture. 2008; 28(4):568-73.

[19] Yaggie JA, McGregor SJ. Effects of isokinetic ankle fatigue on the maintenance of balance and postural limits. Archives of Physical Medicine and Rehabilitation. 2002; 83(2):224-28.

[20] McNair PJ, Heine PJ. Trunk proprioception: enhancement through lumbar bracing. Archives of Physical Medicine and Rehabilitation. 1999; 80(1):96-99.

[21] Forestier N, Teasdale N, Nougier V. Alteration of the position sense at the ankle induced by muscular fatigue in humans. Medicine and Science in Sports and Exercise. 2002; 34(1):117-22.

[22] Yaggie J, Armstrong W. Effects of fatigue on indices of balance. Journal of Sport Rehabilitation. 2004;13:312-22.

[23] Ribeiro F, Mota J, Oliveira J. Effect of exercise-induced fatigue on position sense of the knee in the elderly. European Journal of Applied Physiology. 2007; 99(4):379-85.

[24] Boucher JA, Abboud J, Descarreaux M. The influence of acute back muscle fatigue and fatigue recovery on trunk 
sensorimotor control. Journal of Manipulative and Physiological Therapeutics. 2012; 35(9):662-68.

[25] Sajjadi E, Olyeai G, Talebian S, Hadian M, Jalaei S, Mahmoudi R, et al. [The effect of muscular fatigue on cervical joint position sense in young and healthy men and women: a preliminary study (Persian)]. Modern Rehabilitation. 2014; 8(1):43-51.

[26] Givoni NJ, Pham T, Allen TJ, Proske U. The effect of quadriceps muscle fatigue on position matching at the knee. Journal of Physiology. 2007; 584(1):111-19. 